\title{
REDES EMPRESARIALES EN EL SECTOR TURISMO Y SERVICIOS PARA LA MEJORA DE COMPETITIVIDAD EN CIUDAD JUÁREZ, CHIHUAHUA, MÉXICO: CASO PARQUE CENTRAL HERMANOS ESCOBAR Y PYMES ALEDAÑAS
}

\author{
Jesús Alberto Urrutia de la Garza \\ Tomás Jesús Cuevas Contreras \\ Universidad Autónoma de Ciudad Juárez. México
}

\section{RESUMEN}

La presente investigación, muestra la cooperación y relación que existe entre las pequeñas y medianas empresas (Pymes) del sector turismo y servicios circunvecinos al Parque Central Hermanos Escobar (PCHE) en Ciudad Juárez, Chihuahua (CJS), y su colaboración con este último. Se destacan las relaciones derivadas entre empresas y actores de la zona bajo el marco de la competitividad. En los resultados se presenta la red informal existente, así como la red que se generaría si estas empresas trabajaran bajo condiciones de cooperación, coordinación e intercambio de información y clientes entre los actores involucrados. También, se destaca la iniciativa empresarial para la creación de una red que formalice las relaciones, pero una falta de liderazgo para dirigirla, así como un distanciamiento con el gobierno. Sin embargo, se perciben oportunidades para potenciar las capacidades de aprendizaje e innovación, la reproducción de iniciativas empresariales exitosas y la conformación de redes.

Palabras clave: Redes Empresariales; Pymes; Competitividad; Parque Central Hermanos Escobar; Ciudad Juárez; Chihuahua; México.

Recibido: 1 de diciembre de 2014

Devuelto para su revisión: 11 de mayo de 2015

Aceptado: 15 de junio de 2015

Instituto de Ciencias Sociales y Administración. Universidad Autónoma de Ciudad Juárez. Av. Universidad y Av. Heroico Colegio Militar S/N Zona Chamizal. C.P. 323000, Ciudad Juárez, CHIHUAHUA (México). E-mail: jesus.urrutia@uacj.mx, tccunam@gmail.com 


\title{
Business networks in tourism and service sectors for competitiveness improvement in Ciudad Juarez, Chihuahua, Mexico. a case study: Central Park Hermanos Escobar and surrounding sme's
}

\begin{abstract}
This research shows the relationship and cooperation between small and medium enterprises (SMEs) in the tourism sector and services, surrounding Central Park Hermanos Escobar (PCHE) in Ciudad Juarez, Chihuahua (CJS), and collaboration with the latter. Derived relationships between companies and stakeholders in the area under the framework of competitiveness are emphasized. The results shows the existing informal network and the network that would be generated if these companies work under conditions of cooperation, coordination and information exchange between customers and stakeholders. Entrepreneurship to the creation of a formalized network relationships, but a lack of leadership to direct, and a rift with the government is also argued. However, opportunities are perceived to enhance the capabilities of learning and innovation, replication of successful entrepreneurship and the creation of networks.
\end{abstract}

Keywords: Enterprise Networks; SMEs; Competitiveness; Central Park Hermanos Escobar; Ciudad Juárez; Chihuahua; México.

\section{INTRODUCCIÓN}

En la actualidad, la globalización y los avances tecnológicos han dado lugar a nuevas y mejores tendencias en estructuras productivas, cuya dinámica puede visualizarse en torno a un mayor dinamismo e interacción entre las empresas y los sectores productivos. Esto permite lograr mayores niveles de eficiencia, productividad y competitividad, siendo este último término un tema de estudio, en conjunto con las Pymes, debido a la cantidad de las mismas y a su notable contribución al empleo y Producto Interno Bruto (PIB) de los países, así como al desarrollo de las regiones y ciudades. En el caso de México, la mayoría de las Pequeñas y medianas empresas (Pymes) muestran un bajo crecimiento, o en el peor de los escenarios una tendencia a desaparecer al poco tiempo de su creación, debido a distintos factores que no son apropiadamente gestionados y que no han sido sobre llevados adecuadamente.

Una de las distintas expresiones en los cambios de las estructuras productivas, se percibe en las empresas del sector turismo y servicios, en donde se ha experimentado una evolución importante debido al incremento de las expectativas de los clientes, así como la diversificación de necesidades, gustos y preferencias entre otras razones por una mayor oferta así como un mayor acceso a información de diversos lugares y servicios. Por lo que en este contexto, surge el interés por investigar las Pymes de servicios y turismo que se encuentran alrededor del Parque Central Hermanos Escobar, $(\mathrm{PCHE})$ en materia de redes de colaboración y las relaciones existentes o derivadas entre los distintos actores, en el marco del desarrollo productivo, a través de un estudio exploratorio. 
Por otra parte en las últimas décadas, las redes han sido objeto de estudio por diversos autores que las han abordado desde diversos ángulos (desarrollo productivo, desarrollo local, desarrollo endógeno, entre otros). Por tanto, el articulo presenta una revisión de planteamientos de redes, con la finalidad de enriquecer la visión de la empresa del sector servicios y de los empresarios participantes en este tipo de negocios, pues son las redes una opción de mejora competitiva, dando origen a múltiples relaciones, tanto internas como externas, que constituirían su fortaleza o su debilidad, dependiendo de la forma de dirigirlas y canalizarlas en beneficio de todos los interesados, especialmente en materia de innovación.

\section{ANTECEDENTES}

En este apartado, se presentan hallazgos encontrados de anteriores investigaciones que hacen referencia en las redes empresariales en los que se exponen los beneficios de la asociatividad para el mejoramiento de los procesos empresariales, pero también muestran como en la cultura latinoamericana todavía no existe una adhesión importante hacia esta estrategia de trabajo colectivo entre las empresas. Suárez (2004), plantea la importancia que tiene la estrategia asociativa como una forma viable para acceder al financiamiento por parte de las pequeñas y medianas empresas, además de identificar la necesidad de las empresas de obtener capital de trabajo para invertir en materia prima, tecnologías así como para emplear mano de obra, entre otros. Por otra parte, Ramos (2000), que expone que las Pequeñas y Medianas Empresas se preocupan por su producción y dedica muy poco tiempo a la administración por lo que exhibe, la necesidad de agruparse o asociarse con otros para poder manejar la complejidad de las actividades que se presentan en su operación del día a día, las llevándolas a considerar el hecho asociativo como una consecuencia de supervivencia en un mundo globalizado.

Por otra parte, Jerez (1998), demuestra la factibilidad de utilizar la asociación como estrategia para facilitar el uso de instrumentos financieros, tecnológicos, de capacitación, comercialización y asistencia técnica; además de ser una alternativa para reducir costos, compartir y reducir riesgos, disponer de información, crecer, penetrar mercado y optimizar los tiempos en las empresas. Por otra parte, Perdomo (1998), muestra la importancia que tiene la información como ventaja competitiva para la toma de decisiones en las empresas además de afirmar la insuficiencia de conocimiento sobre las características del ambiente competitivo en el cual se desenvuelven las empresas, factor indispensable para la toma de decisiones eficaces.

En base a lo anteriormente expuesto y tomando en cuenta la situación actual de globalización y constantes cambios que se viven en el ambiente empresarial, los anteriores párrafos dejan en relieve la necesidad de unir esfuerzos para permanecer en el mercado, ya que de lo contrario se corre el riesgo de desaparecer, por lo cual es imperante la búsqueda y propuesta de estrategias para la supervivencia y el desarrollo de las empresas.

\section{CONTEXTUALIZACIÓN Y PLANTEAMIENTO DEL PROBLEMA}

Hoy en día los requerimientos del consumidor han cambiado y aumentado atribuyéndole este fenómeno a una influencia de mercados globalizados, crecimiento de la oferta, diferenciación, especialización, servicios personalizados, entre otros. Todo esto implica 
que el sistema en su conjunto intente renovarse para integrar cambios forzados por esta dinámica que se vive y de esta manera satisfacer la demanda y además procurar ser competitivo. Tal es el caso de las Pymes prestadoras de servicios aledañas al PCHE en Ciudad Juárez, Chihuahua, México (CJS) las cuales muestran a través de evidencias empíricas que enfrentan grandes y variados problemas, que a su vez se traducen en desafíos.

Por otra parte y haciendo referencia a CJS, es de relevancia mencionar que a lo largo de su historia se ha conformado como una ciudad fronteriza dinámica, complicada, pero con prometedoras perspectivas, de tal manera que los datos del INEGI (2010), la determinan como la segunda frontera en orden de importancia económica para México y Estados Unidos. Es tal su actividad, que en la actualidad, la competitividad se ha convertido en un requerimiento para las empresas apostadas en la región.

Con base a la información anterior «es relevante para hacer una investigación científica a las empresas anteriormente mencionadas, dada su ubicación y espacio en el cual se desarrollan» (Barajas, 2009). Luego entonces, la presente investigación, se enfoca en la competitividad por medio de redes de colaboración y cooperación de Pymes partiendo de la perspectiva que faltan de estrategias para captar el mercado que asiste a este lugar público.

La Figura 1, define la ubicación del área de estudio en el centro de la mancha urbana de CJS, destacando la importancia del mismo como el segundo parque público de mayor extensión territorial en la ciudad (solo después del Chamizal), además de ser el único en su tipo ya que dentro de sus instalaciones se realizan constantemente actividades físicas, culturales, sociales, educativas, entre otras debido a que dentro del mismo cuenta con museos, gimnasio, lagos, albercas con toboganes, área de zoológico, jardín botánico, área de juegos infantiles, biblioteca y que además se encuentra en la zona binacional conformada por tres estados en dos países; Chihuahua por parte de México, Texas y Nuevo México por Estados Unidos. «En el año de 1906, el ingeniero Numa P. Escobar fundó la Escuela de Agricultura de Ciudad Juárez, sin embargo la escuela desapareció en 1996 y en sus propiedades se estableció este parque que actualmente es uno de los más bellos de la ciudad». (Moreno, 2013).

En este contexto se plantea el problema para las Pymes que rodean este parque, los cuales muestran desventajas competitivas, con base a las necesidades económicas, geográficas, políticas, sociales y tecnológicas propias de la ciudad, y de esta zona tan importante para la misma. Aunado a lo anterior, estas empresas situadas alrededor del PCHE adquieren mayor relevancia, ya que es el único lugar en su tipo que existe en la ciudad.

Por otra parte, la gestión en el aspecto Redes definidas por (Vázquez, 1999) como «el sistema de relaciones y/o contactos que vinculan a las empresas/actores entre sí, cuyo contenido puede referirse a bienes materiales, información o tecnología» elevaría el nivel de competitividad en las mismas. Por lo cual, el documento propone una aproximación conceptual de variables como cooperación, alianzas estratégicas, asociatividad y colaboración en el marco de las redes empresariales como un factor determinante de competitividad en las anteriormente mencionadas. La investigación cualitativa realizada recoge información que posibilita evaluar la actual manera de trabajar así como la disposición que existe para conformar una red de cooperación y colaboración, como también las competencias claves que tiene o debería de tener la esta zona de la ciudad para fomentar que las Pymes anteriormente mencionadas destaquen positivamente; de aquí se determinan las relaciones críticas que podrían tener configuradas para movilizar recursos. 


\section{Figura 1 \\ UBICACIÓN DEL PARQUE CENTRAL HERMANOS ESCOBAR}

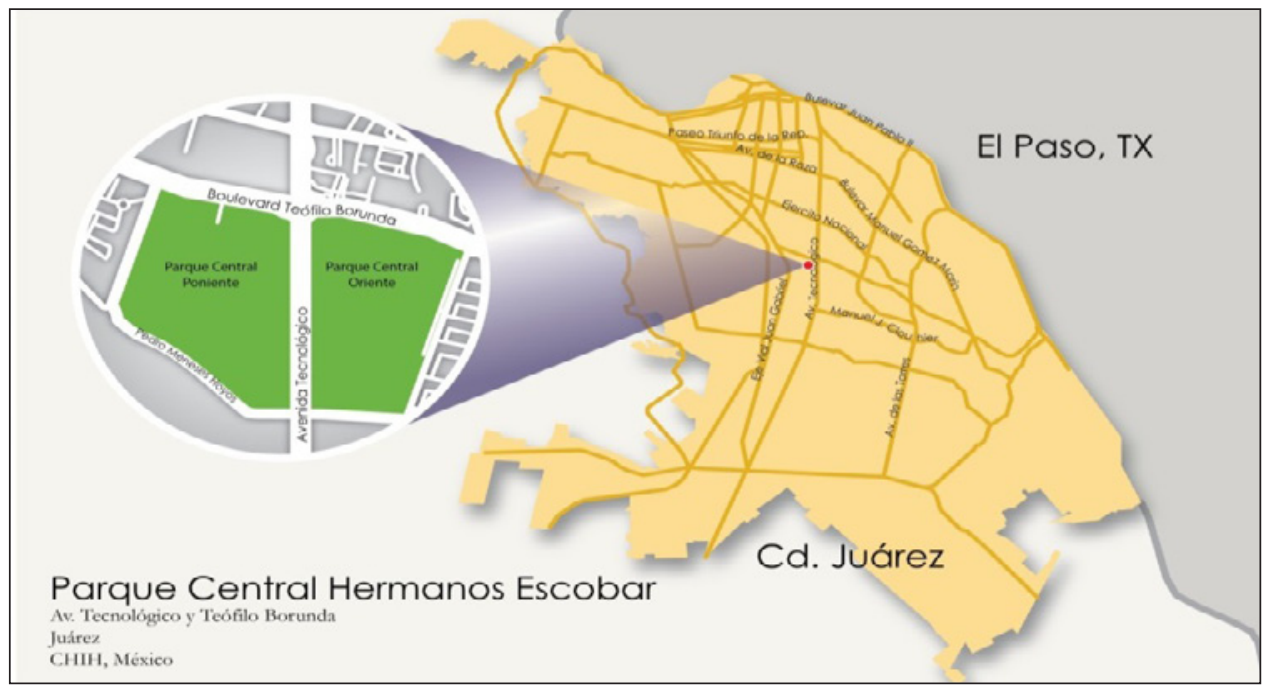

Fuente: elaboración propia en base a Google Maps 2014.

Por tanto, el manuscrito tiene como objetivo primordial examinar si las variables cooperación y colaboración entre estas empresas representan un factor determinante en la competitividad de la zona. Además de objetivo específico, explorar las redes informales que se presentan en la zona; así como, identificar la disposición de las empresa para el desarrollo de una red formal de colaboración; interpretando la misma como una oportunidad para la obtención de ventajas competitivas.

De manera que surgen las interrogantes ¿las variables cooperación y colaboración en este tipo de empresas, son determinantes para el crecimiento y expansión?; ¿La infraestructura en la zona PCHE es un factor reticular para la competitividad de las Pymes?; ¿Las Pymes que se encuentran en zonas de acceso al parque son más competitivas que las que se encuentran en zonas de no acceso? En este sentido, es deseable destacar que la aproximación al fenómeno será con base no solo desde la administración sino desde el ámbito de las consideraciones socioculturales y económicas.

\section{BASES TEÓRICAS}

Haciendo referencia al término Asociatividad, Ruiz (2001a), la menciona «como un mecanismo de cooperación entre ambas partes interesadas», y para el caso de las empresas que se asocian, «mantienen su independencia jurídica y autonomía gerencial, decidiendo voluntariamente realizar esfuerzos conjuntos para la búsqueda de un objetivo común.». Por tanto, la asociatividad en la empresa, es un medio que les permite afrontar la incertidumbre en ambientes de constante cambio e inestabilidad a través de la creación y desarrollo de estrategias que les faciliten competir en igualdad de condiciones reali- 
zando un esfuerzo colectivo para solucionar problemas comunes. Al respecto, Perego (2003a) afirma que:

[...] El término asociatividad surge como uno de los mecanismos de cooperación entre las empresas pequeñas y medianas que están enfrentando un proceso de globalización de las economías nacionales. La globalización económica está redefiniendo los procesos de manufactura al localizar las fábricas en diferentes partes del mundo, abriendo oportunidades pero también significando amenazas para las Pymes, las cuales además de verse presionadas a cambiar sus paradigmas gerenciales requieren diseñar nuevos mecanismos de interrelación con el entorno.

Como toda estrategia empresarial, la asociatividad tiene la característica el ser colectiva y voluntaria pero también implica riesgos que menciona el mismo autor Perego (2003b), «se puede establecer para múltiples propósitos desde el financiamiento hasta la investigación conjunta de determinado problema y, al mismo tiempo, abarcar las diferentes etapas de los procesos básicos de las empresas, a saber, diseño, manufactura, comercialización, servicio post-venta, entre otros pero si no se gestionan estos procesos alguna de las partes puede salir más beneficiada y la otra más afectada.» Por otra parte, (Guillen, 2009) menciona que «La Dirección Estratégica concibe a las redes interorganizativas como un conjunto de organizaciones vinculadas a través de relaciones de intercambio». Por lo que en base a lo expuesto anteriormente, se determina que las características de la asociatividad le otorgan a este mecanismo de cooperación una alta flexibilidad para su aplicación y consecuente operación. Porque:

Es bien sabido que a menos que las pequeñas empresas a desarrollar redes de colaboración con otras empresas, habrá pocas posibilidades de que sean capaces de adaptarse a las condiciones volátiles y alcanzar el desarrollo sostenible. En este contexto, el papel de las asociaciones de turismo es crucial en la prestación de apoyo a las empresas, especialmente las más pequeñas, en la creación de proyectos sostenibles con el medio ambiente de colaboración (Erkuş-Öztürk \& Eraydın, 2010: 120).

Por tanto y en base a (Ruiz, 2001), las principales características de la asociatividad se resumen en los siguientes incisos:

a) Permite resolver problemas conjuntos manteniendo la autonomía gerencial de las empresas participantes

b) La manera de emplear los recursos o beneficios obtenidos a partir de la asociatividad es de la incumbencia exclusiva de cada empresa.

c) Las empresas no están obligados a compartir información que estimen confidencial para sus compañías, como en el caso de la cooperación compulsiva de las redes verticales, o las exigencias de las alianzas estratégicas.

d) Se puede establecer para múltiples propósitos desde el financiamiento hasta la investigación conjunta de determinado problema y, al mismo tiempo, abarcar las diferentes etapas de los procesos básicos de las empresas, a saber: diseño, manufactura, comercialización, servicio post-venta, entre otros. 
e) Le otorga un mecanismo de cooperación inter-empresas una alta flexibilidad de afiliación, operación y ámbito de acción que puede ser empleado tanto por empresas insertadas en redes verticales u horizontales, o incluso para aquéllas que no pertenezcan a ninguna red.

Para el caso de las Redes Empresariales, (López, 2003), las menciona como una alianza estratégica permanente entre un grupo de empresas independientes, que colaboran para alcanzar, objetivos comunes de mediano y largo plazo, orientados hacia el desarrollo de la competitividad de los distintos participantes. De estos existen las redes horizontales o consorcios, (entre 5 y 20 empresas que operan en el mismo ámbito productivo y que generalmente están ubicadas en la misma región) y las redes verticales o desarrollo de proveedores y/o clientes, (refiriéndose a los programas de encadenamiento entre grandes empresas clientes y pequeños proveedores, para incrementar la eficiencia de los mecanismos de proveeduría o distribución). Por otra parte también nombra los Clusters un ambito donde se encuentra una concentración de productores de una misma rama, además de proveedores, comercializadores, asociaciones gremiales, organismos e instituciones de apoyo al sector.

Según la dirección estratégica, «las redes empresariales son un conjunto de organizaciones vinculadas a través de relaciones de intercambio» (García y Medina, 1998a). Las empresas trabajan en conjunto para comercializar sus productos, adquirir insumos, dotarse de servicios comunes, organizarse para producción, especializándose y complementándose entre sí. Además continua (García y Medina, 1998b) «los límites de una red pueden ser establecidos por el propio investigador (planteamiento nominalista) o pueden ser construidos socialmente por las propias organizaciones implicadas (planteamiento realista). En base a estos argumentos, las redes pueden ser utilizadas en la academia para describir las relaciones interorganizativas además de ser percibidas como estratégicas a las que tienen acceso las organizaciones para mejorar su posición competitiva.

Existe la decisión de incorporarse o no a las redes por parte de la empresa. Según Porter (1985) «las estrategias competitivas son aquellas conductas adoptadas por las organizaciones como respuestas a las condiciones de su entorno para lograr alcanzar sus objetivos en situaciones coyunturales del mercado.» Por lo cual las redes empresariales como estrategias de cooperación asienten a los tomadores de decisiones a sumar capacidades para generar condiciones que de manera individual no pudieran lograrse, además de acciones de intercambiar y compartir de fuentes de información, personal, proveedores de materia prima o equipos, consultores, bancos, transportadores, distribuidores o publicistas entre otros.

Por otra parte, las alianzas son otra estrategia empresarial para la mejora de la competitividad y logro de objetivos en las que se ven involucradas las redes; estas se definen como la asociación entre empresas, ya sea de concesión de licencias, acuerdos de abastecimiento, iniciativas de capital de riesgo, adquisiciones conjuntas y muchas otras formas de cooperación, que tienen como objetivo eliminar o reducir en un grado significativo la confrontación entre competidores, proveedores, clientes, entre otros.

Al respecto, (Jiménez, 2000) afirma que «una colaboración mejora las habilidades internas y las tecnologías, y paralelamente, evita que se transfieran a otros las ventajas competitivas, especialmente cuando el otro es un socio ambicioso». También afirma que 
«la alianza entre organizaciones debe verse como una forma de compartir riesgos y fortalezas.» Lo que expresa una necesidad de equilibrio en las recompensas y peligros para las empresas involucradas, que obliga a las empresas a orientar sus esfuerzos en a aprender nuevas destrezas para tener nuevos y mejores productos que a su vez se convierten en ventajas competitivas a mejores costos.

Obviamente, este tipo de colaboración es más fructífera en la medida en que las partes buscan nutrir sus relaciones de negocio en lugar de buscar el control. Una alianza de bajo riesgo es aquella en la que las partes o empresas crean un producto o valor que aisladamente no podrían generar. En muchos casos la alianza estratégica con el tiempo, puede ser el preámbulo de una fusión o de la aparición de un nuevo competidor. Una verdadera alianza debe provocar una permanente evolución de las posibilidades, habilidades, Know how o de las ventajas compartidas.

Un requisito implícito para crear alianzas, es el conocimiento y la empatía entre los dirigentes de las mismas empresas, así como del sector y el mercado. Por lo que se plantea una necesidad de nuevas alternativas, así como cambios en los paradigmas culturales en referencia al trabajo individual. Como puede observarse, la realidad que día a día enfrentan las pymes las obliga a buscar nuevas formas de organización en la búsqueda por sobrevivir en un entorno en el cual la capacidad de aprovechar oportunidades puede representar la diferencia entre fracasar o triunfar.

\section{METODOLOGÍA}

El proyecto es un primer inicio de una investigación que presenta una ventana de oportunidad para el mejoramiento, a través de un estudio cuanticualitativo. El documento se divide en dos secciones. 1) Trabajo de gabinete que sirva de base conceptual, 2) Trabajo de campo. La primera muestra antecedentes y revisión de literatura referente a los términos asociatividad, redes empresariales y alianzas en las Pymes; mientras que en la segunda parte, se proyectan dos mapas del PCHE, con un perímetro de 500 metros a la redonda, en donde se muestran y realizan análisis de ocho Pymes del sector turismo y servicios así como tres hoteles de franquicia y un museo público, con respecto a su ubicación en la zona y sus relaciones con entre estos. Para ello se tomaron como factores de medición, las variables cooperación y colaboración dentro de estas empresas realizando un análisis de la disposición de trabajar en conjunto, así como la ubicación con respecto al PCHE como ventaja competitiva. El artículo se basa en información primaria, proveniente de un instrumento aplicado a los empresarios y gerentes de las diversas empresas alrededor de este espacio público, tomando en consideración las condiciones de cooperación, colaboración o asociación entre actores así como las relaciones existentes en la búsqueda de nuevas posibilidades para así lograr niveles de competitividad capaces de garantizar el éxito en una red en este sector.

La Figura 2. Muestra el área de estudio a través de un croquis del PCHE y las empresas de estudio que se encuentran establecidas a 500 metros del mismo, tomando como referencia el no tener que utilizar ningún medio de transporte para acudir del parque a estos establecimientos. También se puede identificar la Central de Autobuses, ya que también es un referente en el sector turismo para la ciudad. 


\section{Figura 2 CROQUIS DEL PARQUE CENTRAL}

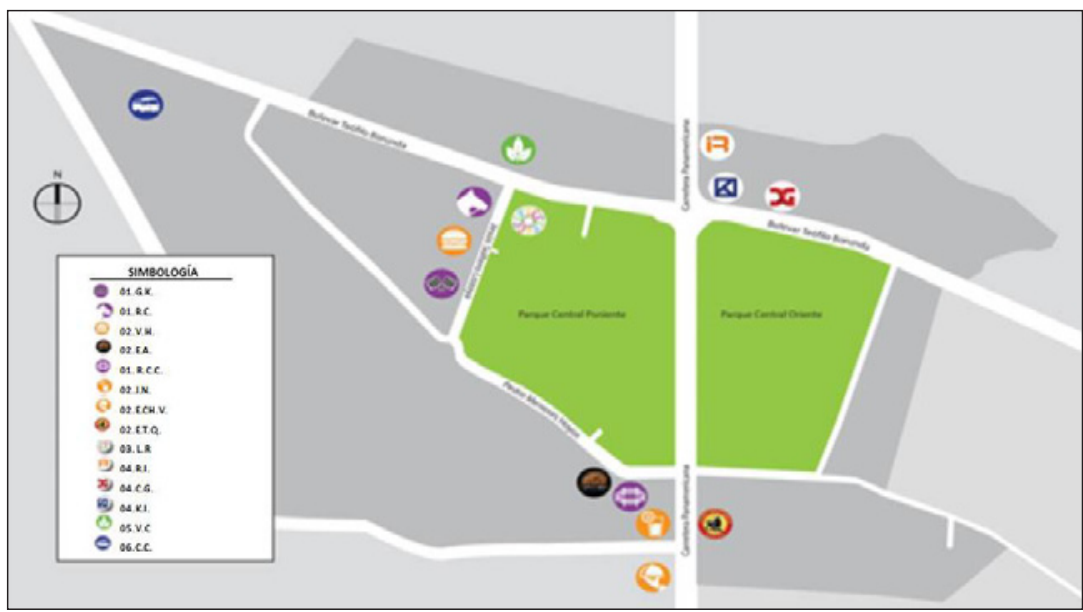

Fuente: elaboración propia en base a Google Maps 2014.

La Tabla 1. Muestra las empresas que rodean al PCHE así como el giro al que pertenecen. En esta, se pueden observar trece empresas que lo rodean, de las cuales cinco son restaurantes, tres hoteles, tres de diversión y un vivero.

\section{Tabla 1}

\section{EMPRESAS QUE RODEAN AL PARQUE CENTRAL PONIENTE}

\begin{tabular}{|l|l|c|}
\hline \multicolumn{1}{|c|}{ Empresa } & \multicolumn{1}{|c|}{ Dirección } & \multicolumn{1}{|c|}{ Giro } \\
\hline 01.G.K. & Jesús Soltero Lozoya S/N Colonia Partido Iglesias & $\begin{array}{c}\text { 01 Diversión y } \\
\text { entretenimiento }\end{array}$ \\
\hline 01.R.C. & Jesús Soltero Lozoya S/N Colonia Partido Iglesias & $\begin{array}{c}\text { 01 Diversión y } \\
\text { entretenimiento }\end{array}$ \\
\hline 02.V.H. & Jesús Soltero Lozoya S/N Colonia Partido Iglesias & 02 Restaurant \\
\hline 02.E.A. & Pedro Meneses Hoyos 6754, a un costado del Gimnasio de Bachilleres & 02 Restaurant \\
\hline 01. R.C.C. & Pedro Meneses Hoyos, a un costado del Gimnasio de Bachilleres & $\begin{array}{c}\text { 01 Diversión y } \\
\text { entretenimiento }\end{array}$ \\
\hline 02.J.N. & Av. Tecnológico 5055 Col La Cuesta CP 32650 & 02 Restaurant \\
\hline 02.E.CH.V. & Carr. Panamericana 5021 Km 12 Cd. Juárez, Chih. & 02 Restaurant \\
\hline 02.E.T.Q. & Panamericana 11. C.P. 32030, Juárez, Chihuahua, México & 02 Restaurant \\
\hline 03. L.R & Av. Teófilo Borunda 6632, Colonia Parque Central Poniente & 03 Museo \\
\hline 04.R.I. & Blvd. Teófilo Borunda No. 6941, Col. Partido Iglesias, CP.32617 Ciudad & 04 Hotel \\
\hline 04.C.G. & Juárez, Chihuahua, México. & 04 Hotel \\
\hline 04.K.I. & Avenida Tecnológico 3750, Partido Iglesias, 32528 Juárez, Chihuahua & 04 Hotel \\
\hline 05.V.C & Teófilo Borunda S/N & 05 Venta de plantas \\
\hline
\end{tabular}

Fuente: elaboración propia en base a trabajo de campo (2014). 


\section{RESULTADOS Y DISCUSIÓN}

Los resultados aquí descritos, se distribuyen en dos secciones principalmente; I) percepción de influencia de PCHE para las empresas aledañas del sector servicios y turismo; II) asociatividad y cooperación para creación de redes empresariales. En primera instancia, se destacan los resultados de la aplicación del instrumento para después presentar la disposición por parte de las empresas a trabajar en conjunto. Como se menciona en la metodología, el documento presenta un examen de las relaciones que tienen las Pymes que se tomaron como muestra para el estudio.

La primera pregunta del instrumento hizo referencia a la relación entre las visitas al parque y a las empresas aledañas, de la cual tan solo el $27 \%$ respondió que sí había una clara correspondencia entre la afluencia del parque central y las personas que visitan su empresa. Con esto se demostró que para algunas empresas aledañas al parque (G.K., V.H. y R.C, ubicadas en la entrada principal), las visitas a éste resulta un beneficio real, por lo que se puede hablar entonces, de una dependencia existente entre ambas en cuanto a los clientes. Por otra parte, el 55\% (principalmente los restaurantes) señalan que sólo en algunas ocasiones como cuando hay graduaciones y eventos; y tan sólo el 18\% (principalmente los hoteles) le es indiferente, ya que no existe una correlación entre las visitas del Parque y sus clientes.

La pregunta dos, cuestionó sobre la percepción de los empresarios en referencia a la influencia que tiene el PCHE sobre sus negocios, para la cual se utilizó la escala positivo, negativo o indistinto. El $73 \%$ de los gerentes y dueños, perciben y aceptan que el PCHE ejerce una influencia positiva sobre sus empresas, ya que comentan que cuando hay muchos visitantes (por cualquier asunto como eventos, esparcimiento u otro), sus negocios se ven claramente beneficiados; sin embargo, a pesar de las ventajas que pudieran obtenerse por la cercanía de los establecimientos, el $27 \%$ señaló que dicha influencia les es indistinta.

La siguiente pregunta del instrumento, buscaba determinar la relación entre los gerentes y dueños de las empresas aledañas con el administrador del PCHE, en donde se encontró, que solo el $18 \%$ de los gerentes de estos negocios conocen a la persona que administra el Parque Central. Paradójicamente y en contraste con las respuestas anteriores obtenidas de los gerentes, en la cuales mencionan una importancia de este lugar para sus empresas, el desinterés por parte del empresario en relacionarse con los tomadores de decisiones del PCHE para el beneficio de su negocio es evidente, ya que el $82 \%$ de éstos menciona no conocer por lo menos al administrador del parque.

Por otra parte, con la pregunta cuatro se obtuvo información con respecto a la colaboración de los gerentes como negocio con actividades del PCHE, con lo cual fue posible demostrar, que independientemente del conocimiento que tengan sobre el entorno o la correlación entre visitantes, el $73 \%$ de los negocios nunca ha participado de alguna manera con las actividades del Parque Central. La Figura 3 muestra el caso de los que respondieron que sí han participado en alguna actividad con el PCHE, la pregunta cinco abordó el tipo de actividad.

La Figura 3, demuestra que la colaboración, se concentran en tres actividades: participación como empresa con venta de sus productos en eventos 33.3\%, participación campañas de reforestación entre otras $33.3 \%$ y otro tipo de participaciones $33.3 \%$. Por tanto, es posible decirse que la colaboración entre los negocios y el parque es casi nula, 
ya que si dichos porcentajes son trasladados en números, tan sólo tres establecimientos han participado y de manera muy esporádica.

\section{Figura 3 \\ TIPO DE ACTIVIDAD EN LA QUE HAN COLABORADO CON EL PARQUE CENTRAL}

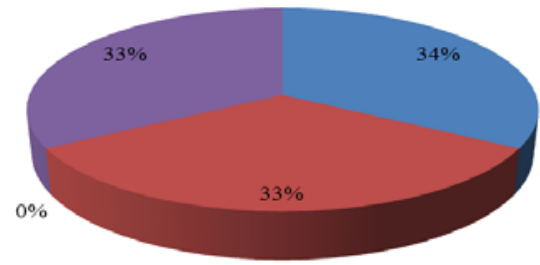

$=$ Eventos

= Campañas

$=$ Promociones

$=$ Otros

Fuente: elaboración propia con base a trabajo de campo 2014.

Sin embargo, también se les cuestionó a los gerentes y dueños sobre la disposición de participar económicamente con el PCHE, a lo que éstos respondieron en un $91 \%$ que efectivamente sí tenían la disposición para participar activamente a manera de lograr mayor competitividad para la zona. Sólo una empresa hotelera equivalente al $9 \%$ restante se mostró renuente, debido a que consideran que su mercado es muy distinto a las personas que acuden al parque, por lo que piensan no necesitar algún tipo de alianza para atraer a esos visitantes, sin embargo apuntó como observación que estaría dispuesto a participar en una red con el fin de no quedarse fuera de las decisiones tomadas.

Luego entonces, dado que la mayoría mostró disposición para colaborar con el PCHE, se les preguntó además cuáles son aquellas actividades en las que estarían dispuestos a participar con el parque, esto con el fin de trabajar en red por parte de los tomadores de decisiones, lo cual no sólo resulta beneficioso en cuestión de clientes, sino que además abre un poco más el panorama en referencia a las posibles estrategias a seguir para la mejora de la competitividad de la zona. La Figura 4 muestra los resultados obtenidos.

\section{Figura 4 \\ ACTIVIDADES CON PRINCIPAL DISPOSICIÓN DE COLABORAR CON EL PARQUE CENTRAL}

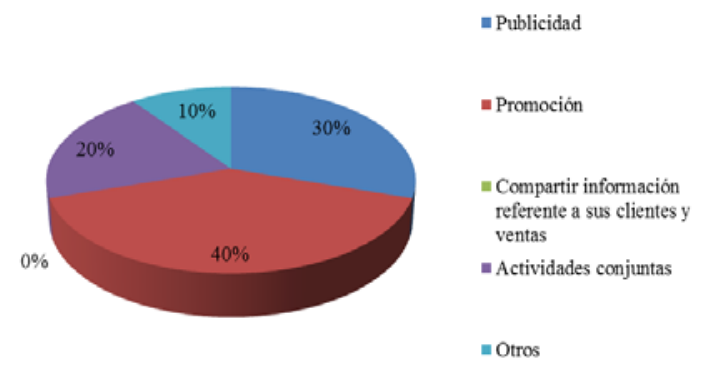

Fuente: elaboración propia con base a trabajo de campo 2014 
La Figura 3, demuestra como las promociones con un $40 \%$ son el principal interés de trabajar en red, mientras que la publicidad con un $30 \%$ quedo en un segundo lugar, seguido por actividades conjuntas para atraer clientes como (eventos deportivos y culturales, degustaciones, ferias, entre otros) con un 20\%; mientras que el $10 \%$ no definió el tipo de actividad. Cabe destacar de esta Figura, que ninguno de los establecimientos aceptó compartir información de sus clientes y ventas, demostrando claramente la cultura individualista de los empresarios. Es coincidente con la postura de Molina (2011): La realidad es un sistema en emergencia permanente, que puede cambiar por la intervención humana organizada y deliberada que persigue una finalidad explicita. Se puede influir en ella redefiniendo relaciones entre los actores, y entre estos y ella, de acuerdo a intereses y lineamientos preestablecidos.

En otro orden de ideas, la segunda sección de estos resultados se presenta en la Figura 5, en la cual se demuestra a través de un diagrama elaborado en base a la investigación de campo, las Pymes que se encuentran aledañas y la situación actual en referencia a colaboración y cooperación en la que se encuentran con respecto a las demás empresas y el parque. En la Figura se muestra la red informal en la cual se interpreta con flechas como ha sido la colaboración (quien ha apoyado a que empresa) y cooperación entre estas y con el PCHE.

Como comentan Cuevas, Carmona y Zizaldra (2012) : Las redes operan no solo dentro del turismo sino también en torno al turismo de las organizaciones formales, entre los participantes en la industria, así como los distintos organismos de gobiernos y la sociedad civil para proporcionar un foro importante para el desarrollo y a comunicación de intereses y estrategias.

\section{Figura 5 \\ SITUACIÓN ACTUAL DE COLABORACIÓN Y COOPERACIÓN EN EL PCHE}

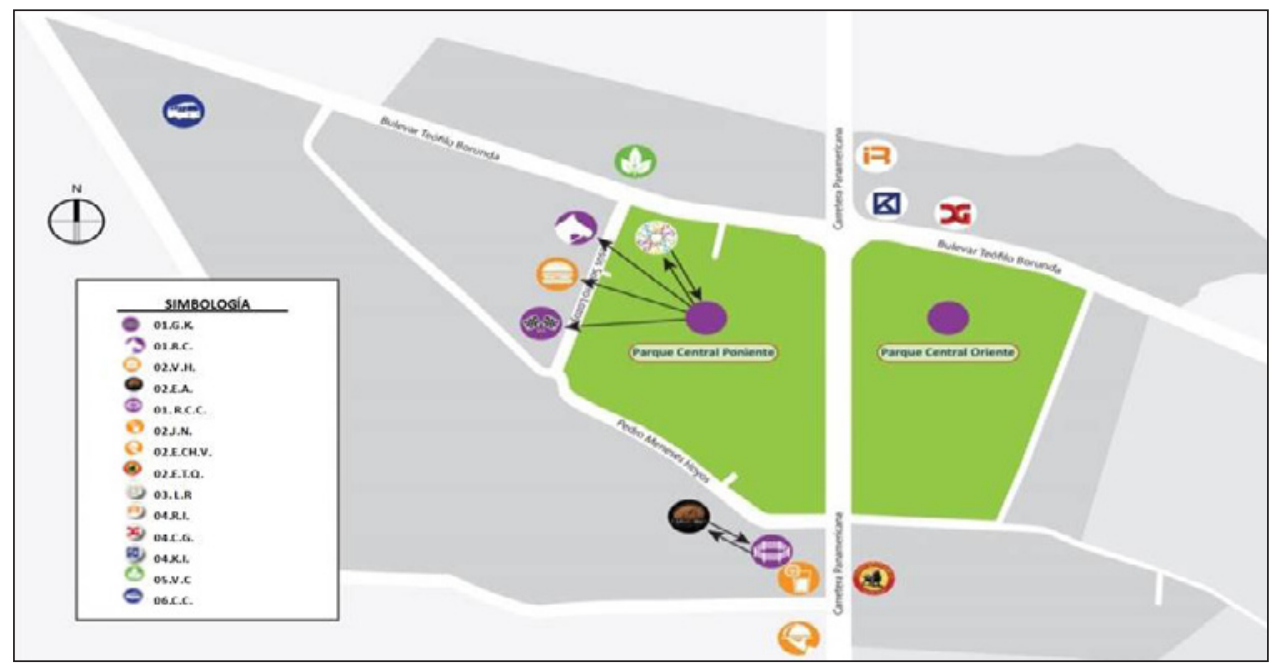

Fuente: elaboración propia en base a trabajo de campo (2014). 
La anterior Figura 5, ilustra para el caso de los negocios que se encuentran en la calle Jesús Soltero Lozoya cómo han recibido apoyo por parte de las oficinas de los directivos del parque en referencia a permitirles hacer promociones con los usuarios dentro de las instalaciones del parque, así mismo comentan el dueño de la empresa G.K. que hay una buena relación con la directora del parque, sin embargo, ella tiene comprometidas sus decisiones con sus superiores, lo cual obstaculiza que se dé una relación comercial.

Por otra parte, dentro del mismo parque se encuentra el Museo la Rodadora, el cual comenta el entrevistado, que existe una coordinación de sus actividades con el Parque Central, sin embargo, las decisiones se toman individuales ya que el museo es administrado por un patronato empresarial (es decir es necesario avisar que tendrán actividades).

Para el caso de los tres hoteles que se encuentran en contra esquina del parque, mencionan no tener ningún vínculo con la gerencia del mismo, lo que se percibe como un oportunidad para prestarle un servicio complementario a sus clientes. Los restaurantes E.A., J.N, E.CH.V, y el T. Q., mencionan no tener ningún vínculo con el Parque Central, sin embargo, el en caso de E.CH.V y el T. Q. mencionaron que su mercado en ocasiones son los clientes del Parque Central; es decir, que van de visita al parque y llegan a comer con ellos.

En el caso del restaurante E. A. mencionó que su segmento de mercado no es el mismo que asiste al Parque Central, por lo que nunca ha tenido la necesidad de buscar una alianza o asociación con el parque; sin embargo, percibió una necesidad en la ciudad así como en su restaurante, por lo cual el 16 de Septiembre del 2013 inició con un negocio de carreras de caballos, rodeo, entre otros, mismo que comenta lo ha unido a las estrategias del restaurante y obtenido muy buenos beneficios.

\section{Figura 6 \\ INTENCIONES DE COLABORACIÓN Y COOPERACIÓN EN EL PCHE}

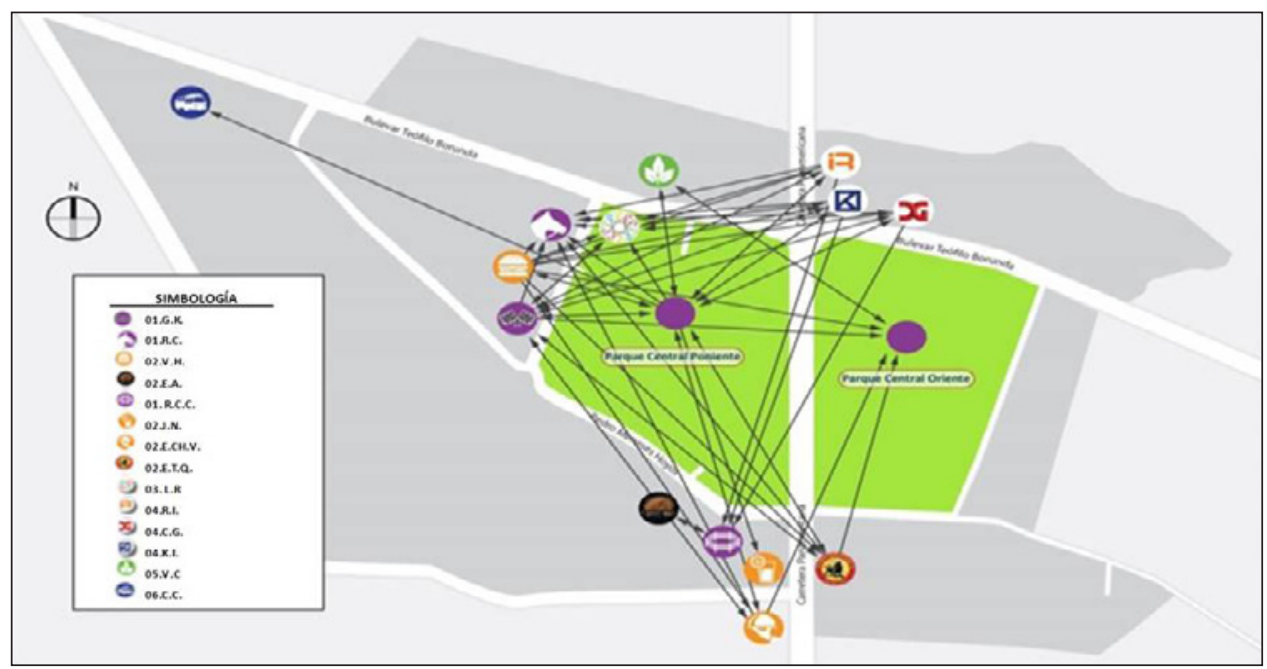

Fuente: elaboración propia en base a trabajo de campo (2014). 
Haciendo referencia al negocio de V.J (venta de jugos), que se encuentra sobre la carretera Panamericana o Ave. Tecnológico, se destaca que el Parque Central tiene muchísimos visitantes que van a practicar algún tipo de actividad física, sin embargo, su ubicación no le permite captar este mercado. Menciona que su mercado son los automóviles que pasan sobre la Avenida, más no los que visitan el parque y buscan una bebida sana y natural.

Para el caso de la disposición que hay para trabajar en conjunto, la Figura 9, presenta una visión esquemática de una red basada en las respuestas obtenidas en el instrumento; mismas que demuestran que si todas las empresas trabajaran bajo un criterio de colaboración y cooperación pretendiendo llegar a un mismo fin establecido se pudiera dar la creación de una red formal.

Cabe señalar, que hay muy buena disposición e intención de trabajar en conjunto, solo que se percibe una falta de liderazgo además de apatía para dirigir la red por parte de los empresarios, además que el instrumento dejo en relieve una mala actitud hacia el gobierno por parte de los empresarios, los cuales pretenden que el parque les solucione en gran parte sus problemas.

\section{CONCLUSIONES}

El manuscrito tiene un carácter exploratorio. Si bien, no era ahondar en el tema de la competitividad dentro de las Pymes que rodean el PCHE, si se pretende examinarla desde la óptica de las redes. Su propósito es una primera aproximación entre la gestión de la asociatividad y su vínculo natural con la competitividad y los factores que presenta esta zona de la ciudad. Sin embargo se llega a una primera conclusión, el desarrollo y mejora de las Pymes en esta zona se ve comprometido, si bien por una serie de factores, pero que destaca la falta de comunicación, y liderazgo entre los empresarios, además de infraestructura en la zona ya la dispersión de Pymes no concuerda con los accesos al parque.

Aunque, las empresas evaluadas, se encuentran establecidas por periodos considerados lagos de tiempo en sus establecimientos. Las derivaciones apuntan a una mejor planeación para el aprovechamiento de los visitantes del PCHE como posibles clientes además de la utilización de la infraestructura de este al máximo. Por otra parte, existe la disposición de trabajar en conjunto por parte de los empresarios, lo que se convertiría en un factor dinamizador de la zona.

Luego entonces la incorporación de estos factores en la planeación y el trabajo en conjunto de las Pymes a través de herramientas para la constante medición en los mismos los haría más controlables, optimaría la competitividad de la zona, que a su vez se traduce en oportunidades para su aproximación a mas mercado tanto local, como también el mercado internacional, (ya que el parque reporta mucho visitante de la ciudad de El Paso Texas); ya que actualmente se presenta una inexactitud en la incorporación de estrategias de trabajo en redes, lo que en el corto plazo perjudica la competitividad al empresario Pyme, posiblemente por falta de conocimiento del contexto sociocultural y económicoadministrativo. «En conclusión, la competitividad en general y la competitividad turística en particular es un concepto complejo sobre el que existe un amplio y productivo debate académico. Esta complejidad alcanza su punto culminante cuando se incorporan aspectos territoriales en los análisis de competitividad de los destinos turísticos» (Perles, 2014) 
En definitiva se requiere profundizar en el tema y promover nuevas investigaciones, para revelar especificidades y necesidades de las Pymes. «Un marco ancho, es decir, un abanico más completo de referencias, que pasaría por la consideración de índices de precios u otros indicadores al uso, permitiría obtener una visión más robusta» (Perles, 2014); lo que ayudaría a debatir, el turismo como motor económico mexicano y sus oportunidades de inclusión de valor, temáticas que quedan por el momento fuera del alcance del presente documento.

\section{REFERENCIAS}

BARAJAS, M. DEL R. (2009): Cuatro décadas del modelo maquilador en el norte de México. México: COLEF. [En línea]. http://www.colef.mx/jorgecarrillo/wp-content/ uploads/2012/04/PU345.pdf

CUEVAS CONTRERAS, T.; CARMONA VEGA, E. y ZIZALDRA HERNÁNDEZ, I. (2012): Turismo Transfronterizo Reticular. Exploración del caso como Ciudades Hermanas: Ciudad Juárez, Chihuahua - El Paso, Texas. En Turismo en la frontera del conocimiento: entre la modernidad y la postmodernidad. (Eds) Cuevas, T. y Palafox, A. México: Universidad Autónoma de Ciudad Juárez / Academia Mexicana de Investigación Turística, A.C., pp. 75-95.

ERKUŞ-ÖZTÜRK, H., y ERAYDIN, A. (2010): «Environmental governance for sustainable tourism development: Collaborative networks and organisation building in the Antalya tourism region». Tourism Management, vol. 31(1), pp. 113-124.

MEDINA MUÑOZ, D.R. y GARCÍA FALCÓN, J.M. (1998): «Enfoques teóricos sobre las relaciones interorganizativas: una revisión comparativa». Revista Europea de Dirección y Economía de la Empresa, no 7 (3), pp. 72-103.

GUILLEN, A., RODRIGUEZ, J. y VEGA, Y. (2009): «Redes Empresariales, alternativa de desarrollo gerencial para Pymes del sector plástico del Estado Lara. Gestion y Gerencia, vol. 3 n ${ }^{\circ}$, Barquisimeto, Venezuela, pp. 4-27

INSTITUTO NACIONAL DE GEOGRAFÍA Y ESTADÍSTICA. INEGI. (2010): Espacio y datos de México. Disponible en: http://www3.inegi.org.mx/sistemas/mapa/visualizador/Default.aspx

JEREZ, J. (1998): La Asociación como Estrategia para que las Pequeñas y Medianas Empresas del estado Trujillo alcancen una mayor Productividad y Competencia: un estudio exploratorio situacional. Tesis de Grado, Universidad de los Andes. Mérida.

JIMÉNEZ A.R. (2000): Las alianzas estratégicas como gestión empresarial: El caso de México. http://ciberconta.unizar.es/LECCION/alianza/INICIO.HTML. (Noviembre de 2004).

LÓPEZ, CERDÁN C. (2003): Redes empresariales: Experiencia en la región andina.

MORENO SANCHEZ, E.C. (2014): Configuración de los espacios abiertos en Ciudad Juárez. Pacarina del Sur. [En línea], año 4, núm. 15.

PERDOMO, A. (1998): Uso Estratégico de la Información como Ventaja Competitiva para la Toma de Decisiones en las PyMEs. Tesis de Maestría en Administración Financiera, Decanato de Administración y Contaduría (DAC) Universidad Centroccidental Lisandro Alvarado (UCLA). 
PEREGO, L. H. (2003): La asociatividad como estrategia competitiva. [En línea], http:// www.eumed.net/cursecon/libreria/lhp/1e.htm.

PERLES RIBES J.F., RAMÓN RODRUIGUEZ A.B. y SEVILLA JIMENEZ, M. (2014): «La cuota de mercado como indicador de competitividad en los destinos turísticos: sentido y limitaciones. Cuadernos de Turismo, n 34, pp. 265-285.

PORTER, M. (1985): Competitive Advantage: creating and superior performance with a new introduction. New York: Free Press; London Collier.

RAMOS, N. (2000). La Asociación como Estrategia Financiera de las Empresas del Sector Metalmecánico de las Zonas Industriales de Barquisimeto, estado Lara. Tesis de maestría en Gerencia Financiera, Universidad Centroccidental Lisandro Alvarado (UCLA).

RUIZ, A. F. (2001): La Asociatividad en las PYMEs. http://www.gestiopolis.com/canales/ economia/articulos/20/asociatividad.htm. (Noviembre de 2004)

SUÁREZ, J. (2004): La Estrategia Asociativa como una forma para acceder al Financiamiento: Pequeñas y Medianas Empresas del Sector Téxtil. Barquisimeto, Estado Lara. Tesis de maestría en Gerencia Financiera, Decanato de Administración y Contaduría (DAC) Universidad Centroccidental Lisandro Alvarado (UCLA).

MOLINA, S. (2011): Turismo Creativo. El fin de la competitividad. Chile Editores . CL. VÁZQUEZ BARQUERO, A. (1999): «Dinámica productiva y desarrollo urbano. La respuesta de la ciudad de Vitoria a los desafíos de la globalización». Revista Eure, Vol. $\mathrm{XXV}, \mathrm{n}^{\circ}$ 74, pp. 19-33.

\section{Entrevistas personales}

CALVO E., entrevista personal, 02 de octubre 2014. La Rodadora

CANIZALES E., entrevista personal, 25 de septiembre 2014. El Arrancadero

INURRETA F., entrevista personal, 15 de octubre 2014. Real Inn

LOPEZ V., entrevista personal, 01 de octubre 2014. Venta Hamburguesas

URRUTIA J., entrevista personal, 03 de octubre 2014. Renta de Caballos

VALLES C., entrevista personal, 29 de septiembre 2014. Chaparral de Valles

ZETINA R., entrevista personal, 29 de septiembre 2014. Go karts 